\title{
RELAXATION OF EULER EQUATIONS AND HYDRODYNAMIC INSTABILITIES
}

\author{
BY \\ JEAN DUCHON (La Plaine-St. Sauveur, 38160 St. Marcellin, France) \\ AND \\ RAOUL ROBERT (21, Avenue Plaine Fleurie, 38240 Meylan, France)
}

\begin{abstract}
We present a relaxed version of incompressible Euler equations that permits foliated flows involving two velocities. These relaxed equations allow a twophase evolution of some vortex sheets as an alternative to discontinuous solutions of Euler equations. In the case of two perfect fluids of different densities superposed one over the other, we show that this relaxation process yields a linearly well-posed two-phase solution.
\end{abstract}

1. Introduction. We consider the following Cauchy problem for Euler equations, giving the motion of an incompressible perfect fluid:

$$
\begin{aligned}
& \mathbf{u}_{t}+(\mathbf{u} \cdot \nabla) \mathbf{u}=-\nabla p \\
& \operatorname{div} \mathbf{u}=0 \\
& \mathbf{u}(0, x)=\mathbf{u}_{0}(x)
\end{aligned}
$$

where $\mathbf{u}(t, x)$ is the velocity field of the fluid at time $t, x \in \mathbf{R}^{3}$, and $p$ is the normalized pressure. More precisely, we are interested in vortex sheets dynamics; that is, when the singular part of curl $\mathbf{u}_{0}$ is a measure carried by a regular surface. In the particular case where the regular part of curl $\mathbf{u}_{0}$ is zero, we deduce from (E) the Birkhoff-Rott system giving the evolution of the sheet [3, 22]. We know that the Cauchy problem for that system is ill posed in Hadamard's sense (linearly and nonlinearly, see $[6,13])$, the terms of spatial frequency $\xi$ in the initial data being amplified by a factor $e^{|\xi| t}$. This phenomenon is usually known as Kelvin-Helmholtz instability.

Then if we follow Hadamard's modelling principle, which says that systems coming from physical modelling have to be well posed, the Birkhoff-Rott system for vortex sheets is not correct and must be discarded.

If we model, in a correct way, a physical phenomenon, we expect to get a wellposed system. In the case of vortex sheets, it seems that the inaccurate constraint 
lies in the assumption that the singular vorticity is at each time concentrated on a regular surface.

Nevertheless, if we follow Ovsjannikov's ideas and make use of scales of analytic functions, we can find an analytic solution during a finite time for any analytic initial data [5, 22]. But the physical meaning of analytic solutions is not obvious, and this point must be accurately discussed. As we shall see later, strong arguments indicate that analytic solutions can appear as "nonentropic" (or nonphysical) ones even before the appearance of a singularity, which generally occurs in a finite time.

What can we do then?

The first idea is to come back to Euler equations, written in conservative form (we replace the term $(\mathbf{u} \cdot \nabla) \mathbf{u}$ by $\partial_{k}\left(\mathbf{u}_{k} \mathbf{u}\right)$ ), and to define weak solutions, that is, nonregular velocity fields satisfying the system in the distribution sense. Of course, the problem is to find a weak solution with a vortex sheet initial data. This has been investigated by Di Perna and Majda [10]. The existence of a weak solution to the Cauchy problem, in the case of a compactly supported and positive initial vorticity distribution, was recently proved by Delort [24].

Another approach is to relax the Euler system, following the ideas developed by L. C. Young on the calculus of variations [23]. Relaxing Euler equations consists in defining generalized flows which will be solutions of an extended form of Euler equations. This is a complex program in general, and we present here a first step in this method, restricting ourselves to the case of foliated flows involving two velocities. In this case, by means of homogenization techniques, we can establish a closed system of equations governing the evolution of the foliated flow. This relaxed system, which we shall call the foliated Euler system (F.E), is canonically determined since we suppose that the microscopic structure of the flow is regularly organized in thin sheets sliding freely one over the other.

Our purpose in this paper is first to introduce the system (F.E), and then to show that this system admits remarkable particular solutions:

-We exhibit a foliated flow, solution of (F.E), continuous for all $t>0$, and admitting for initial data a discontinuous stationary solution of (E).

-In the case of two perfect fluids of different densities, we consider the initial state where the heavier is superposed over the lighter with horizontal interface and velocities equal to zero. Then we show that there is a two-phase solution as an alternative to the trivial steady one (corresponding to an ill-posed problem for the interface). We can show that the linearized system about this solution gives a wellposed Cauchy problem.

Some authors have defined measure-valued solutions for hyperbolic systems [9, 20]. Notice that we work here with very particular measure-valued solutions; this permits us to make explicit the conditions coming from the microscopic structure of the flow, and to set up a closed set of equations. We refer also to [19], where the question of the convection of microstructures is investigated by means of homogenization methods. Finally, let us notice that generalized flows have been introduced, from a Lagrangian point of view, in [4], using a relaxed version of the least action principle. Our approach is essentially different since we are mainly interested here in the Cauchy 
problem. As we shall see in the comments of Sect. 4 , this relaxation process raises a lot of questions.

2. The foliated Euler system. In this section, we set up the system of equations giving the evolution of an incompressible foliated flow of a perfect fluid.

At a microscopic level, we can view a foliated flow as a foliation of thin sheets of two perfect fluids $F^{+}$and $F^{-}$, with the same density, sliding freely one over the other with two different velocities.

The macroscopic description of a foliated flow is a set of unknown functions defined on $[0, T] \times \Omega$, where $T>0$ is a given number and $\Omega$ is an open subset of $\mathbf{R}^{3}$.

There are three sorts of conditions that the unknown functions will have to satisfy:

(i) cinematic conditions,

(ii) conservation conditions,

(iii) dynamic conditions.

(i) Cinematic conditions. Here we define a foliated velocity field. A foliated velocity field is a set of four functions $\left(\mathbf{u}^{+}, \mathbf{u}^{-}, \mathbf{n}, v\right)$, where:

$-\mathbf{u}^{+}(t, x), \mathbf{u}^{-}(t, x)$ are two velocity fields,

- $\mathbf{n}(t, x)$ is a unit vector field giving the direction normal to the sheets, and

$-v(t, x)$ is a scalar function giving the normal speed of the sheets.

These functions must satisfy the following conditions:

(*) For all $t$, in the neighborhood of every point $x, \mathbf{n}(t, x)$ is the normal field to a family of surfaces (which we shall call the sheets). This can be expressed by the classical Frobenius condition $\mathbf{n} \cdot \operatorname{curl} \mathbf{n}=0$ [1].

$(* *)$ At time $t$ and position $x$, the normal speeds of $F^{+}$and $F^{-}$are equal to the normal speed of the sheets, $v(t, x)$ :

$$
\mathbf{u}^{+} \cdot \mathbf{n}=\mathbf{u}^{-} \cdot \mathbf{n}=v .
$$

$(* * *)$ The sheets are material surfaces; that is, they are transported by the velocity field $v \mathbf{n}(t, x)$. This can be expressed by the following equation:

$$
\mathbf{n}_{t}=-P_{n}[v(\mathbf{n} \cdot \nabla) \mathbf{n}+\nabla v],
$$

where the lower index $t$ indicates differentiation with respect to $t$ and $P_{n}$ is the orthonogal projection onto the plane normal to $\mathbf{n}$.

This expression is a consequence of the following technical lemma.

Lemma 1. Let $\mathbf{n}(t, x)$ be a unit vector field (of course $\mathbf{n}$ is supposed to be smooth enough). We suppose that, for all $t, \mathbf{n}$ is, locally in $x$, the normal field to a family of surfaces (we shall say that $\mathbf{n}$ is locally integrable) transported by the velocity field $\mathbf{V}(t, x)=v(t, x) \mathbf{n}(t, x)$. Then we have

$$
\mathbf{n}_{t}=-P_{n}[v(\mathbf{n} \cdot \nabla) \mathbf{n}+\nabla v] .
$$

Proof. Let us define the flow $\Phi(t, x)$ associated to the field $\mathbf{V}(t, x)$ by

$$
\frac{\partial}{\partial t} \boldsymbol{\Phi}(t, x)=\mathbf{V}(t, \Phi(t, x)), \quad \boldsymbol{\Phi}(0, x)=x .
$$


Let $\Gamma$ be a surface which is normal to the field $\mathbf{n}(0, x)$, and going through the point $x$. Let us write that $\mathbf{n}(t, \Phi(t, x))$ is normal to $\Phi(t, \Gamma)$ at the point $\Phi(t, x)$ :

$$
\mathbf{n}(t, \Phi(t, x)) \cdot D \Phi(t, x)[\tau]=0
$$

for all vectors $\tau$ tangent to $\Gamma$ at $x$. Here $D \Phi(t, x)$ denotes the differential of $\Phi(t, x)$ with respect to the variable $x$.

If we differentiate this last expression with respect to $t$, we get

$$
\begin{aligned}
& {\left[n_{i t}(t, \Phi(t, x))+\frac{\partial n_{i}}{\partial y_{k}}(t, \Phi(t, x)) \frac{\partial \Phi_{k}}{\partial t}(t, x)\right] \frac{\partial \Phi_{i}}{\partial x_{j}} \tau_{j}} \\
& \quad+n_{i}(t, \Phi(t, x)) \frac{\partial^{2} \Phi_{i}}{\partial x_{j} \partial t}(t, x) \tau_{j}=0 .
\end{aligned}
$$

Note that

$$
\frac{\partial^{2} \Phi_{i}}{\partial x_{j} \partial t}(t, x)=\frac{\partial}{\partial x_{j}} V_{i}(t, \Phi(t, x))=\frac{\partial V_{i}}{\partial y_{k}}(t, \Phi(t, x)) \frac{\partial \Phi_{k}}{\partial x_{j}}(t, x) ;
$$

then the above relation gives, for $t=0$,

$$
\left[n_{i t}+\frac{\partial n_{i}}{\partial y_{k}} V_{k}\right] \tau_{i}+n_{i} \frac{\partial V_{i}}{\partial y_{j}} \tau_{j}=0
$$

As $\mathbf{V}=v \mathbf{n}$, this gives $\left(\mathbf{n}_{t}+v(\mathbf{n} \cdot \nabla) \mathbf{n}+\nabla v\right) \cdot \tau=0$, using the relation $\mathbf{n} \cdot \partial \mathbf{n} / \partial y_{j}=0$.

Moreover, we have $\mathbf{n}_{t} \cdot \mathbf{n}=0$. We finally get

$$
\mathbf{n}_{t}=-P_{n}[v(\mathbf{n} \cdot \nabla) \mathbf{n}+\nabla v] .
$$

For the converse, it suffices to notice that if $\mathbf{n}$ is a locally integrable field satisfying the above relation with a given function $v(t, x)$, then the images by the flow $\Phi$ (associated to the velocity field $v \mathbf{n}$ ) of the normal surfaces at time $t=0$ are the normal surfaces at time $t$.

In short, a foliated velocity field is a set of functions $\mathbf{u}^{+}, \mathbf{u}^{-}, \mathbf{n}, v$ defined on $[0, T] \times \Omega$, and satisfying:

$$
\begin{gathered}
\mathbf{n} \cdot \operatorname{curl} \mathbf{n}=0, \\
\mathbf{u}^{+} \cdot \mathbf{n}=v, \quad \mathbf{u}^{-} \cdot \mathbf{n}=v, \\
\mathbf{n}_{t}=-P_{n}[v(\mathbf{n} \cdot \nabla) \mathbf{n}+\nabla v] .
\end{gathered}
$$

Notice here that if the relation $(*)$ is satisfied for $t=0$, then $(* *)$ and $(* * *)$ imply that it is satisfied for all time.

To settle the conservation and dynamic equations, we use classical homogenization techniques.

Let us consider a sequence $\mathbf{u}^{\varepsilon}(t, x)$ of solutions of the incompressible Euler equations which is bounded in the space $\mathbf{L}^{\infty}([0, T] \times \Omega)$ :

$$
\mathbf{u}_{t}^{\varepsilon}+\partial_{k}\left(\mathbf{u}_{k}^{\varepsilon} \mathbf{u}^{\varepsilon}\right)=-\nabla p^{\varepsilon}, \quad \operatorname{div} \mathbf{u}^{\varepsilon}=0
$$

We suppose that we can separate two phases $F^{+}$and $F^{-}$by means of a characteristic function $e^{\varepsilon}(t, x): e^{\varepsilon}(t, x)=1$ if at time $t$ the point $x$ is in $F^{+}, e^{\varepsilon}(t, x)=0$ 
if not. Then $e^{\varepsilon}$ satisfies the conservation equation $e_{t}^{\varepsilon}+\operatorname{div}\left(e^{\varepsilon} \mathbf{u}^{\varepsilon}\right)=0$, which traduces the conservation of the phases during the motion.

We suppose now that the sequence $\mathbf{u}^{\varepsilon}$ converges (in a convenient sense which will be defined below) towards a foliated flow, and then deduce the set of equations necessarily satisfied by $\mathbf{u}^{+}, \mathbf{u}^{-}, \mathbf{n}, v$.

Let us state precisely the hypothesis of convergence on the sequence $\mathbf{u}^{\varepsilon}$. We shall use the following notation: To each vector field $\mathbf{u}(t, x)$ defined on $[0, T] \times \Omega$ we associate the Young measure [23] $\delta_{\mathrm{u}}$ defined by

$$
\left\langle\delta_{\mathbf{u}}, \phi\right\rangle=\iint \phi(t, x, \mathbf{u}(t, x)) d x d t
$$

for all continuous compactly supported test functions $\phi(t, x, y)$ on $[0, T] \times \Omega \times \mathbf{R}^{3}$. Then we suppose that there is a smooth function $e(t, x)$ such that the measure $e^{\varepsilon} \delta_{\mathbf{u}^{\varepsilon}}$ converges for the vague topology towards $e \delta_{\mathbf{u}^{+}}$; that is

$$
\left\langle e^{\varepsilon} \delta_{\mathbf{u}^{\varepsilon}}, \phi\right\rangle \rightarrow\left\langle e \delta_{\mathbf{u}^{+}}, \phi\right\rangle \quad \text { for all } \phi,
$$

and similarly $\left(1-e^{\varepsilon}\right) \delta_{\mathbf{u}^{\varepsilon}}$ converges vaguely towards $(1-e) \delta_{\mathbf{u}^{-}}$.

We readily deduce the weak convergences $\sigma\left(\mathbf{L}^{\infty}, \mathbf{L}^{1}\right)$ :

$$
e^{\varepsilon} \rightarrow e, \quad e^{\varepsilon} \mathbf{u}^{\varepsilon} \rightarrow e \mathbf{u}^{+}, \quad \mathbf{u}^{\varepsilon} \rightarrow \overline{\mathbf{u}}=e \mathbf{u}^{+}+(1-e) \mathbf{u}^{-} .
$$

$e(t, x)$ is the local concentration of the phase $F^{+}$.

(ii) Conservation equations. From the weak convergences above, taking the limits in the distribution sense, the equations $e_{t}^{\varepsilon}+\operatorname{div}\left(e^{\varepsilon} \mathbf{u}^{\varepsilon}\right)=0$ and $\operatorname{div} \mathbf{u}^{\varepsilon}=0$ readily give the conservation equations

$$
e_{t}+\operatorname{div}\left(e \mathbf{u}^{+}\right)=0, \quad \operatorname{div}\left(e \mathbf{u}^{+}+(1-e) \mathbf{u}^{-}\right)=0 .
$$

(iii) Dynamic equations. The dynamic equation satisfied by $\mathbf{u}^{\varepsilon}$ can be written as

$$
\operatorname{curl}\left(\mathbf{u}_{t}^{\varepsilon}+\partial_{k}\left(\mathbf{u}_{k}^{\varepsilon} \mathbf{u}^{\varepsilon}\right)\right)=\mathbf{0} .
$$

Writing $\mathbf{u}_{k}^{\varepsilon} \mathbf{u}^{\varepsilon}=e^{\varepsilon} \mathbf{u}_{k}^{\varepsilon} \mathbf{u}^{\varepsilon}+\left(1-e^{\varepsilon}\right) \mathbf{u}_{k}^{\varepsilon} \mathbf{u}^{\varepsilon}$, we see that the convergence hypothesis implies that $\mathbf{u}_{k}^{\varepsilon} \mathbf{u}^{\varepsilon} \rightarrow e \mathbf{u}_{k}^{+} \mathbf{u}^{+}+(1-e) \mathbf{u}_{k}^{-} \mathbf{u}^{-}$) (weakly), from which we get the limit in the distribution sense:

$$
\operatorname{curl}\left(\overline{\mathbf{u}}_{t}+\partial_{k}\left(e \mathbf{u}_{k}^{+} \mathbf{u}^{+}+(1-e) \mathbf{u}_{k}^{-} \mathbf{u}^{-}\right)\right)=\mathbf{0} .
$$

Introducing the average pressure $p(t, x)$ and the notation $[\mathbf{u}]=\mathbf{u}^{+}-\mathbf{u}^{-}$, we finally get

$$
\overline{\mathbf{u}}_{t}+\partial_{k}\left(\overline{\mathbf{u}}_{k} \overline{\mathbf{u}}\right)+\partial_{k}\left(e(1-e)[\mathbf{u}]_{k}[\mathbf{u}]\right)=-\nabla p
$$

the expression which shows the Reynolds tensor $e(1-e)[\mathbf{u}]_{k}[\mathbf{u}]_{i}$.

At this point, we have 11 unknown scalar functions: $\mathbf{u}^{+}, \mathbf{u}^{-}, \mathbf{n}, v, e, p$, and only nine scalar equations: the above dynamical equation, the two conservation equations, and the cinematic equations $(* *)$ and $(* * *)$.

To obtain the two dynamical equations that will close the system, we make a hypothesis of dynamical foliation on the convergence of $\nabla p^{\varepsilon}$. Heuristically, we 
must have, at the limit, only one pressure gradient in the direction of the sheets; this traduces the fact that the pressure is continuous through the microscopic sheets.

We obviously have $\nabla p^{\varepsilon} \rightarrow \nabla p$ in the distribution sense. We may state our hypothesis in writing that for all vector fields $\tau(t, x)$, tangent to the sheets (i.e., satisfying $\tau \cdot \mathbf{n}=0$ ), the sequence $\nabla p^{\varepsilon} \cdot \tau$ converges strongly in some functional space towards $\nabla p \cdot \tau$. In fact, it is more convenient to suppose directly that

$$
e^{\varepsilon} \nabla p^{\varepsilon} \cdot \tau \rightarrow e \nabla p \cdot \tau
$$

and

$$
\left(1-e^{\varepsilon}\right) \nabla p^{\varepsilon} \cdot \tau \rightarrow(1-e) \nabla p \cdot \tau,
$$

the convergences being taken in the distribution sense.

Denoting $\gamma^{\varepsilon}=\mathbf{u}_{t}^{\varepsilon}+\partial_{k}\left(\mathbf{u}_{k}^{\varepsilon} \mathbf{u}^{\varepsilon}\right)$, and using the conservation equation $e_{t}^{\varepsilon}+\operatorname{div}\left(e^{\varepsilon} \mathbf{u}^{\varepsilon}\right)=$ 0 , we get

$$
e^{\varepsilon} \gamma^{\varepsilon}=\left(e^{\varepsilon} \mathbf{u}^{\varepsilon}\right)_{t}+\partial_{k}\left(e^{\varepsilon} \mathbf{u}_{k}^{\varepsilon} \mathbf{u}^{\varepsilon}\right)
$$

it follows that $e^{\varepsilon} \gamma^{\varepsilon} \rightarrow e \gamma^{+}$in the distribution sense, $\gamma^{+}=\mathbf{u}_{t}^{+}+\left(\mathbf{u}^{+} \cdot \nabla\right) \mathbf{u}^{+}$.

But $e^{\varepsilon} \gamma^{\varepsilon} \cdot \tau \rightarrow-e \nabla p \cdot \tau$, and we deduce that $\gamma^{+} \cdot \tau=-\nabla p \cdot \tau$; similarly, we have $\gamma^{-} \cdot \tau=-\nabla p \cdot \tau$.

Of course, it suffices to write these relations for two independent tangent vector fields $\tau_{1}$ and $\tau_{2}$; this gives four new scalar equations, but they are not independent of the dynamical equation which can be written as $e \gamma^{+}+(1-e) \gamma^{-}=-\nabla p$. In the sequel, we only retain the relation $\gamma^{+} \cdot \tau=\gamma^{-} \cdot \tau$ for all tangent vector fields $\tau$. This can also be written as

$$
\left(\gamma^{+}-\gamma^{-}\right) \wedge \mathbf{n}=\mathbf{0}
$$

Let us resume the complete set of equations which govern the evolution of the foliated flow:

$$
\begin{aligned}
& \text { (1) } \mathbf{u}^{+} \cdot \mathbf{n}=v, \\
& \text { (2) } \mathbf{u}^{-} \cdot \mathbf{n}=v, \\
& \text { (3) } \mathbf{n}_{t}=-P_{n}[v(\mathbf{n} \cdot \nabla) \mathbf{n}+\nabla v], \\
& \text { (4) } e_{t}+\operatorname{div}\left(e \mathbf{u}^{+}\right)=0, \\
& \text { (5) } \operatorname{div}\left(e \mathbf{u}^{+}+(1-e) \mathbf{u}^{-}\right)=0, \\
& \text { (6) } e \gamma^{+}+(1-e) \gamma^{-}=-\nabla p, \\
& \text { (7) }\left(\gamma^{+}-\gamma^{-}\right) \wedge \mathbf{n}=\mathbf{0} .
\end{aligned}
$$

These equations are valid inside a foliated region. Now, we have to specify the conditions at the boundary and at the interface between foliated and unfoliated regions.

Conditions at the boundary and at the interface between foliated and unfoliated regions. Suppose that we have a foliated flow in an open subset $\Omega$ of $\mathbf{R}^{3}$. Then, at the boundary $\partial \Omega$, we only have to express that the velocity of each phase $F^{+}, F^{-}$ is tangent to the boundary; that is, $\mathbf{u}^{+} \cdot \nu=\mathbf{u}^{-} \cdot \nu=0$, where $\nu$ denotes the unit normal vector at $\partial \Omega$ (pointing outward). 
Let us now examine the conditions at the interface between an unfoliated region (where the motion of the fluid is governed by ordinary Euler equations) and a foliated region (governed by the system (F.E)). We first notice that the system (F.E) will certainly have a "more hyperbolic" character than (E) since it permits each phase to relax into the other; consequently a foliated region, whose boundary travels at a finite speed, can appear. Since our purpose is to replace, if possible, discontinuous solutions of (E) by continuous foliated solutions of (F.E), we shall require that, at the interface between an unfoliated and a foliated region, the functions $e, \overline{\mathbf{u}}$, and $p$ be continuous (this supposes that $\overline{\mathbf{u}}$ is extended in a natural way by $\mathbf{u}^{+}$or $\mathbf{u}^{-}$in the unfoliated regions).

On the conservation of the kinetic energy. Let $\Omega$ be an open bounded subset of $\mathbf{R}^{3}$. We consider a flow in $\Omega$ which can be foliated in some region, and which satisfies the boundary conditions $\mathbf{u}^{+} \cdot \nu=\mathbf{u}^{-} \cdot \nu=0$.

The total kinetic energy of the fluid is given by

$$
\Xi=\frac{1}{2} \int_{\Omega}\left(e\left|\mathbf{u}^{+}\right|^{2}+(1-e)\left|\mathbf{u}^{-}\right|^{2}\right) d x
$$

We have

Proposition 1. During the motion, we have $d \Xi / d t=0$.

Proof. For the sake of simplicity, we give the proof in the case when $0<e<1$ everywhere. To handle the general case, it suffices to work with the (continuous) extensions by 0 of $e \mathbf{u}^{+}$and $(1-e) \mathbf{u}^{-}$to the whole domain. We have

$$
\frac{d \Xi}{d t}=\frac{1}{2} \int_{\Omega}\left(e \mathbf{u}^{+}\right)_{t} \cdot \mathbf{u}^{+}+e \mathbf{u}^{+} \cdot \mathbf{u}_{t}^{+}+\left((1-e) \mathbf{u}^{-}\right)_{t} \cdot \mathbf{u}^{-}+(1-e) \mathbf{u}^{-} \cdot \mathbf{u}_{t}^{-} d x,
$$

but $\mathbf{u}_{t}^{+}=\gamma^{+}-\left(\mathbf{u}^{+} \cdot \nabla\right) \mathbf{u}^{+}$and $\left(e \mathbf{u}^{+}\right)_{t}=e \gamma^{+}-\partial_{k}\left(e \mathbf{u}_{k}^{+} \mathbf{u}^{+}\right)$, this last equality coming from the relation $e_{t}+\operatorname{div}\left(e \mathbf{u}^{+}\right)=0$.

Of course, we have similar relations for $\mathbf{u}^{-}$; hence

$$
\begin{aligned}
\frac{d \Xi}{d t}= & \int_{\Omega} e \gamma^{+} \cdot \mathbf{u}^{+} d x-\frac{1}{2} \int_{\Omega} \partial_{k}\left(e \mathbf{u}_{k}^{+} \mathbf{u}^{+}\right) \cdot \mathbf{u}^{+}+\left(\left(\mathbf{u}^{+} \cdot \nabla\right) \mathbf{u}^{+}\right) \cdot e \mathbf{u}^{+} d x \\
& + \text { similar terms in } \mathbf{u}^{-} .
\end{aligned}
$$

Noticing that the second integrand can be written as $\operatorname{div}\left(e\left|\mathbf{u}^{+}\right|^{2} \mathbf{u}^{+}\right)$, and that

$$
\int_{\Omega} \operatorname{div}\left(e\left|\mathbf{u}^{+}\right|^{2} \mathbf{u}^{+}\right) d x=\int_{\partial \Omega} e\left|\mathbf{u}^{+}\right|^{2} \mathbf{u}^{+} \cdot \nu d \sigma=0,
$$

we obtain

$$
\frac{d \Xi}{d t}=\int_{\Omega}\left(e \gamma^{+} \cdot \mathbf{u}^{+}+(1-e) \gamma^{-} \cdot \mathbf{u}^{-}\right) d x
$$

Using the relation $\left(\gamma^{+}-\gamma^{-}\right) \cdot\left(\mathbf{u}^{+}-\mathbf{u}^{-}\right)=0$, one verifies that

$$
e \gamma^{+} \cdot \mathbf{u}^{+}+(1-e) \gamma^{-} \cdot \mathbf{u}^{-}=\left(e \mathbf{u}^{+}+(1-e) \mathbf{u}^{-}\right) \cdot\left(e \gamma^{+}+(1-e) \gamma^{-}\right) \text {. }
$$

Then

$$
\frac{d \Xi}{d t}=-\int_{\Omega} \overline{\mathbf{u}} \cdot \nabla p d x=\int_{\Omega} p \operatorname{div} \overline{\mathbf{u}} d x-\int_{\partial \Omega} p \overline{\mathbf{u}} \cdot \nu d \sigma=0
$$


The total kinetic energy splits into two parts.

- The macroscopic kinetic energy:

$$
\Xi_{\mathrm{ma}}=\frac{1}{2} \int_{\Omega}|\overline{\mathbf{u}}|^{2} d x
$$

- The two-phase (or turbulent) kinetic energy:

$$
\Xi_{\mathrm{di}}=\Xi-\Xi_{\mathrm{ma}}=\frac{1}{2} \int_{\Omega} e(1-e)\left(\mathbf{u}^{+}-\mathbf{u}^{-}\right)^{2} d x .
$$

An estimate on the growth of the two-phase kinetic energy. As we have seen, the mean velocity $\overline{\mathbf{u}}$ satisfies

$$
\overline{\mathbf{u}}_{t}+\partial_{k}\left(\overline{\mathbf{u}}_{k} \overline{\mathbf{u}}\right)+\partial_{k}\left(e(1-e)[\mathbf{u}]_{k}[\mathbf{u}]\right)=-\nabla p .
$$

Let us compute

$$
\frac{d \Xi_{\mathrm{ma}}}{d t}=\int_{\Omega} \overline{\mathbf{u}} \cdot \overline{\mathbf{u}}_{t} d x
$$

and

$$
\frac{d \Xi_{\mathrm{ma}}}{d t}=-\int_{\Omega} \nabla p \cdot \overline{\mathbf{u}} d x-\int_{\Omega}(\overline{\mathbf{u}} \cdot \nabla) \overline{\mathbf{u}} \cdot \overline{\mathbf{u}} d x-\int_{\Omega} \partial_{k}\left(e(1-e)[\mathbf{u}]_{k}[\overline{\mathbf{u}}]\right) \cdot \overline{\mathbf{u}} d x .
$$

As $\operatorname{div} \overline{\mathbf{u}}=0, \overline{\mathbf{u}} \cdot n=0$ at the boundary and $(\overline{\mathbf{u}} \cdot \nabla) \overline{\mathbf{u}}=\nabla \overline{\mathbf{u}}^{2} / 2-\overline{\mathbf{u}} \wedge$ curl $\overline{\mathbf{u}}$, applying the divergence formula we see that the two first integrals vanish. Then we get

$$
\frac{d \Xi_{\mathrm{ma}}}{d t}=-\int_{\Omega} \operatorname{div}\left(e(1-e)[\mathbf{u}]_{k}[\mathbf{u}]\right) \overline{\mathbf{u}}_{k} d x=\int_{\Omega} e(1-e)[\mathbf{u}]_{k}[\mathbf{u}] \cdot \nabla \overline{\mathbf{u}}_{k} d x ;
$$

hence

$$
\left|\frac{d \Xi_{\mathrm{ma}}}{d t}\right| \leq C(t) \Xi_{\mathrm{di}}, \quad C(t)=2 \sup _{\substack{0 \leq s \leq t \\ x \in \Omega}} \sum_{k, i}\left|\partial_{k} \overline{\mathbf{u}}_{i}(s, x)\right| .
$$

From the conservation of the total kinetic energy, it follows that

$$
\frac{d \Xi_{\mathrm{ma}}}{d t}=-\frac{d \Xi_{\mathrm{di}}}{d t}
$$

Then

$$
\left|\frac{d \Xi_{\mathrm{di}}}{d t}\right| \leq C(t) \Xi_{\mathrm{di}}
$$

which gives

$$
\Xi_{\mathrm{di}}(t) \leq \Xi_{\mathrm{di}}(0) \exp \left(\int_{0}^{t} C(s) d s\right) .
$$

This estimate is valid for any $C^{1}$ solution (i.e., if $e, e \mathbf{u}^{+},(1-e) \mathbf{u}^{-}$, and $e(1-e)[\mathbf{u}]_{k}[\mathbf{u}]$ are $C^{1}$ functions of $t$ and $x$ on $\left.[0, T] \times \bar{\Omega}\right)$.

As a consequence, we have the following proposition. 
Proposition 2. Let us consider a $C^{1}$ solution of (F.E) such that $\Xi_{\mathrm{di}}(0)=0$ (i.e., the initial data identify with the field $\overline{\mathbf{u}}(0))$. Then we shall have $\Xi_{\mathrm{di}}(t)=0$ all the time and (F.E) identifies with the classical Euler system for the field $\overline{\mathbf{u}}$. So if $\Xi_{\mathrm{di}}(0)=0$ and $\overline{\mathbf{u}}(0, x)$ belongs to the Hölder space $C^{1, \alpha}(\bar{\Omega})$, there is no $C^{1}$ solution of the Cauchy problem for (F.E) other than the classical solution of Euler equations (cf. [2, 17]).

Study of the two-dimensional case. First, we try to find particular solutions of (F.E) such that $\mathbf{u}^{+}, \mathbf{u}^{-}, e$, and $p$ depend only on $x_{1}, x_{2}, \mathbf{u}_{3}^{+}=\mathbf{u}_{3}^{-}=0, \mathbf{n}(t, x)=$ $(0,0,1)$, and $v(t, x)=0$.

Then we have $\gamma^{+}=\gamma^{-}$and the system (F.E) reduces to the simpler form

$$
\begin{aligned}
& e_{t}+\operatorname{div}\left(e \mathbf{u}^{+}\right)=0, \\
& \operatorname{div}\left(e \mathbf{u}^{+}+(1-e) \mathbf{u}^{-}\right)=0, \\
& \gamma^{+}=-\nabla p, \\
& \gamma^{-}=-\nabla p .
\end{aligned}
$$

In fact (F.E') models a two-dimensional foliated flow whose microscopic structure is three dimensional. We recognize here a classical system for mixtures.

Let us suppose now that the microscopic structure is also two dimensional. All the variables only depend on $x_{1}, x_{2}$, and $\mathbf{u}_{3}^{+}=\mathbf{u}_{3}^{-}=\mathbf{n}_{3}=0$. We then have the same system (F.E) but now two-dimensional, the only differences being that the condition $(*)$ is automatically satisfied and Eq. (3) can be put in a simpler form by using the angle $\phi(t, x)$ defined in complex notations by $\mathbf{n}=i e^{i \phi} ;(3)$ then becomes

$$
\phi_{t}=\nabla v \cdot \tau-v \nabla \phi \cdot \mathbf{n},
$$

where $\tau=e^{i \phi}$.

It will be convenient to introduce the functions $\omega$ and $\Lambda$ :

$$
\omega=\operatorname{curl} \overline{\mathbf{u}}, \quad \Lambda=\left(\mathbf{u}^{+}-\mathbf{u}^{-}\right) \cdot \tau .
$$

Equations (3), (4), (6), and (7) then give

$$
\begin{aligned}
& \phi_{t}=\nabla v \cdot \tau-v \nabla \phi \cdot \mathbf{n}, \\
& e_{t}+\operatorname{div}\left(e \mathbf{u}^{+}\right)=0, \\
& \omega_{t}+\overline{\mathbf{u}} \cdot \nabla \omega+\operatorname{curl}\left(\partial_{k}\left(e(1-e) \Lambda^{2} \tau_{k} \tau\right)\right)=0, \\
& \Lambda_{t}+\left(\left(\mathbf{u}^{+} \cdot \nabla\right) \mathbf{u}^{+}-\left(\mathbf{u}^{-} \cdot \nabla\right) \mathbf{u}^{-}\right) \cdot \tau=0 .
\end{aligned}
$$

The third equation is obtained by taking the curl of (6) and the fourth by differentiating $\Lambda=\left(\mathbf{u}^{+}-\mathbf{u}^{-}\right) \cdot \tau$ with respect to $t$ :

$$
\Lambda_{t}=\left(\mathbf{u}_{t}^{+}-\mathbf{u}_{t}^{-}\right) \cdot \tau+\phi_{t}[\mathbf{u}] \cdot \mathbf{n} .
$$

From $\mathbf{u}_{t}^{+}=\gamma^{+}-\left(\mathbf{u}^{+} \cdot \nabla\right) \mathbf{u}^{+}$and $\left(\gamma^{+}-\gamma^{-}\right) \cdot \tau=0$, we get the result. 
We complete (F.E $\left.E^{\prime \prime}\right)$ by the equations

$$
\begin{aligned}
& \mathbf{u}^{+}=\overline{\mathbf{u}}+(1-e) \Lambda \tau, \\
& \mathbf{u}^{-}=\overline{\mathbf{u}}-e \Lambda \tau, \\
& v=\overline{\mathbf{u}} \cdot \mathbf{n}, \\
& \operatorname{curl} \overline{\mathbf{u}}=\omega, \\
& \operatorname{div} \overline{\mathbf{u}}=0 .
\end{aligned}
$$

These equations, together with the boundary conditions on $\partial \Omega$, permit us to determine $\mathbf{u}^{+}, \mathbf{u}^{-}, \overline{\mathbf{u}}$, and $v$ from $\phi, e, \omega$, and $\Lambda$. Thus we are led to a system of four equations with four unknown scalar functions.

REMARK. The chosen approach to set up the system (F.E) permits us to proceed shortly. But a difficult question about the existence of such sequences $\mathbf{u}^{\varepsilon}$ arises. However, as the system is canonical, we shall certainly get the same set of equations whatever approach we choose.

3. Three particular solutions of the foliated Euler system. Apart from the solution of the general Cauchy problem for the system (F.E), we now exhibit three remarkable solutions.

First EXAMPLE. We take $\Omega=\mathbf{R}^{2}$, and let $\alpha$ denote a positive parameter. In the $(x, y)$ plane, we get an $x$-invariant solution by taking

$$
\begin{aligned}
\mathbf{n}(t, x, y)=(1,0), & v(t, x, y) & =0, \\
\mathbf{u}^{+}(t, x, y)=\left(0, y /(3 t)-\alpha t^{-1 / 3}\right), & \mathbf{u}^{-}(t, x, y) & =\left(0, y /(3 t)+\alpha t^{-1 / 3}\right)
\end{aligned}
$$

for all $t>0$ and $|y| \leq 3 \alpha t^{2 / 3}$. We complete the construction by setting $\mathbf{u}^{+}(t, x, y)=$ 0 for $y>3 \alpha t^{2 / 3}$ and $\mathbf{u}^{-}(t, x, y)=0$ for $y<-3 \alpha t^{2 / 3}$.

Taking

$$
e(t, x, y)= \begin{cases}t^{-2 / 3} y /(6 \alpha)+1 / 2 & \text { for }|y| \leq 3 \alpha t^{2 / 3} \\ 1 & \text { for } y>3 \alpha t^{2 / 3}, \\ 0 & \text { for } y<-3 \alpha t^{2 / 3}\end{cases}
$$

the conservation equations (4) and (5) are satisfied. We check that in the foliated region $\left(|y| \leq 3 \alpha t^{2 / 3}\right) \quad \gamma^{+}=\gamma^{-}=-\nabla p$, with $p=y^{2} /\left(9 t^{2}\right) ; p$ can be continuously extended by a constant outside the foliated region. A straightforward computation shows that, per unit length in $x$, the kinetic energy of the system is constant and equal to $2 \alpha^{3}$.

We verify that when $t \rightarrow 0, \mathbf{u}^{+}$and $\mathbf{u}^{-}$converge to 0 in the distribution sense. So the system has for initial data a velocity field equal to zero and a kinetic energy of density $2 \alpha^{3}$, spread along the $x$-axis.

SECOND EXAMPLE. Our second example is an expanding foliated layer which starts as a vortex sheet and asymptotes to a shear layer of finite thickness for large $t$. More precisely, in the $(x, y)$ plane, we shall construct:

(i) A velocity field $\mathbf{u}_{0}(y)$ parallel to the $x$-axis and discontinuous along the $x$-axis (see the profile of $\mathbf{u}_{0}$ in Fig. 1). 


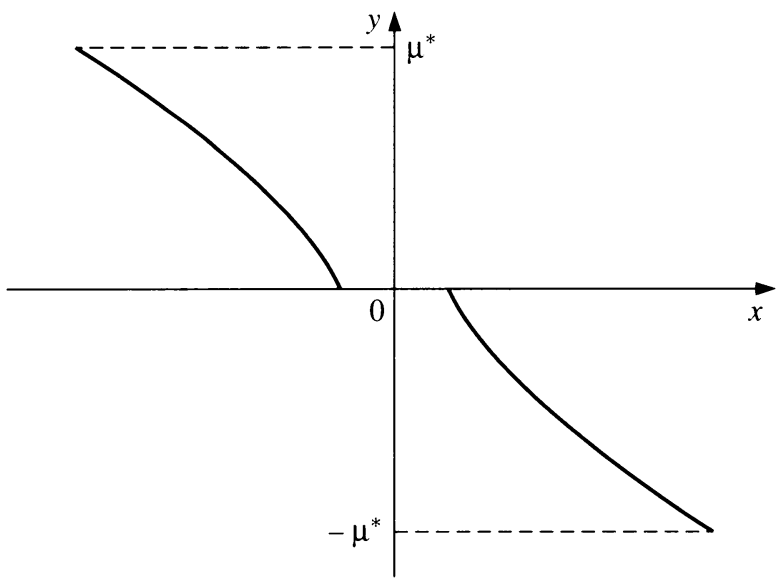

FIG. 1.

(ii) A nonnegative strictly increasing function $\mu(t)$ such that $\mu(0)=0$ and $\lim \mu(t)=\mu^{*}$ (when $t \rightarrow+\infty$ ), where $0<\mu^{*}<+\infty$.

(iii) A foliated flow in the layer $|y| \leq \mu(t)$ which is a solution of (F.E) fitting continuously with $\mathbf{u}_{0}$ at the boundary of the layer. We require also that $e, \overline{\mathbf{u}}$, and $p$ be continuous on the whole layer $|y| \leq \mu^{*}$.

This construction will give a solution of (F.E) in the layer $|y| \leq \mu^{*}$, admitting the velocity field $\mathbf{u}_{0}$ as the initial value. Of course we notice that in the regions where the flow is not foliated (F.E) reduces to the ordinary Euler system.

Here we shall take $\Omega=\left\{(x, y)|| y \mid \leq \mu^{*}\right\}$, ( $\mu^{*}$ will be specified later $)$, and we write (F.E) in the particular case of an $x$-invariant foliated layer. We shall suppose that when the parameter $\alpha$ increases from 0 to 1 , the coordinate $y(t, \alpha)$, defined by $e(t, y(t, \alpha))=\alpha$, increases from the bottom to the top of the layer. At a fixed time $t$, because of the $x$-invariance, all the microscopic sheets are obtained by translating the sheet going through the origin, which is supposed to have the parametric representation $(x(t, \alpha), y(t, \alpha))$. Then we have to handle Eqs. (1)-(7) of (F.E).

Conservation equations (4), (5). Let us define the functions $\mathbf{v}^{+}(t, \alpha)$ and $\mathbf{v}^{-}(t, \alpha)$ by $\mathbf{u}^{ \pm}(t, y(t, \alpha))=\mathbf{v}^{ \pm}(t, \alpha)$. From the equation $e(t, y(t, \alpha))=\alpha$, we deduce $e_{y}=1 / y_{\alpha}$ and $e_{t}=-y_{t} / y_{\alpha}$, where we suppose $y_{\alpha}>0$ for all $t>0$. Since $\operatorname{div}\left(e \mathbf{u}^{+}\right)=\left(e u_{2}^{+}\right)_{y}=\left(\alpha v_{2}^{+}\right)_{\alpha} / y_{\alpha}$, Eq. (4) gives $-y_{t}+\left(\alpha v_{2}^{+}\right)_{\alpha}=0$, from which we get

$$
v_{2}^{+}=Y_{t} / \alpha,
$$

where $Y(t, \alpha)=\int_{0}^{\alpha} y(t, \sigma) d \sigma$.

We seek solutions satisfying $\mathbf{u}^{+}(t,-y)=-\mathbf{u}^{-}(t, y)$. Then we have $y(t, \alpha)+$ $y(t, 1-\alpha)=0$, and this gives

$$
v_{2}^{-}=-Y_{t} /(1-\alpha) \text {. }
$$

Cinematic equations (1), (2), (3). Equation (3) is obviously satisfied since we start here from a given parametrization of the microscopic sheets. 
We have

$$
\mathbf{n}=\left(-y_{\alpha} /\left(x_{\alpha}^{2}+y_{\alpha}^{2}\right)^{1 / 2}, x_{\alpha} /\left(x_{\alpha}^{2}+y_{\alpha}^{2}\right)^{1 / 2}\right)
$$

and

(1) and (2) then give

$$
v=\left(x_{\alpha} y_{t}-x_{t} y_{\alpha}\right) /\left(x_{\alpha}^{2}+y_{\alpha}^{2}\right)^{1 / 2}
$$

$$
\begin{aligned}
& v_{1}^{+}=m\left(v_{2}^{+}-y_{t}\right)+x_{t}, \\
& v_{1}^{-}=m\left(v_{2}^{-}-y_{t}\right)+x_{t},
\end{aligned}
$$

where $m(t, \alpha)=x_{\alpha} / y_{\alpha}$.

Dynamic equation (6). Using $x$-invariance, the equation $e \gamma^{+}+(1-e) \gamma^{-}=-\nabla p$ reduces to

$$
e \gamma_{1}^{+}+(1-e) \gamma_{1}^{-}=0
$$

From the expression $\gamma^{+}=\mathbf{u}_{t}^{+}+\left(\mathbf{u}^{+} \cdot \nabla\right) \mathbf{u}^{+}$, one readily obtains

$$
\begin{aligned}
& \gamma_{1}^{+}(t, \alpha)=v_{1 t}^{+}+v_{1 \alpha}^{+}\left(v_{2}^{+}-y_{t}\right) / y_{\alpha}, \\
& \gamma_{2}^{+}(t, \alpha)=v_{2 t}^{+}+v_{2 \alpha}^{+}\left(v_{2}^{+}-y_{t}\right) / y_{\alpha},
\end{aligned}
$$

and similar expressions for $\gamma_{1}^{-}, \gamma_{2}^{-}$. From formulas (3.1)-(3.4), we get

$$
\begin{gathered}
v_{1 \alpha}^{+}=\left(m_{\alpha}-m / \alpha\right)(Y / \alpha-y)_{t}+y_{\alpha} m_{t}, \\
v_{1 \alpha}^{-}=-\left(m_{\alpha}+m /(1-\alpha)\right)(Y /(1-\alpha)+y)_{t}+y_{\alpha} m_{t},
\end{gathered}
$$

which give

$$
\begin{gathered}
\gamma_{1}^{+}=v_{1 t}^{+}+\left(m_{\alpha}-m / \alpha\right)\left[(Y / \alpha-y)_{t}\right]^{2} / y_{\alpha}+m_{t}(Y / \alpha-y)_{t}, \\
\gamma_{1}^{-}=v_{1 t}^{-}+\left(m_{\alpha}+m /(1-\alpha)\right)\left[(Y /(1-\alpha)+y)_{t}\right]^{2} / y_{\alpha}-m_{t}(Y /(1-\alpha)+y)_{t} .
\end{gathered}
$$

From (3.3) and (3.4), we get

$$
\alpha v_{1}^{+}+(1-\alpha) v_{1}^{-}=x_{t}-m y_{t} .
$$

Finally (3.5) yields the equation

$$
x_{t t}-2 m_{t} y_{t}-m y_{t t}+A m_{\alpha} / y_{\alpha}-B m / y_{\alpha}=0,
$$

where

$$
\begin{aligned}
& A=\alpha\left[(Y / \alpha-y)_{t}\right]^{2}+(1-\alpha)\left[(Y /(1-\alpha)+y)_{t}\right]^{2}, \\
& B=\left[(Y / \alpha-y)_{t}\right]^{2}-\left[(Y /(1-\alpha)+y)_{t}\right]^{2} .
\end{aligned}
$$

Dynamic equation (7). In two dimensions, (7) reduces to $\left(\gamma^{+}-\gamma^{-}\right) \cdot\left(\mathbf{u}^{+}-\mathbf{u}^{-}\right)=0$, that is, $\left(\gamma_{1}^{+}-\gamma_{1}^{-}\right)\left(v_{1}^{+}-v_{1}^{-}\right)+\left(\gamma_{2}^{+}-\gamma_{2}^{-}\right)\left(v_{2}^{+}-v_{2}^{-}\right)=0$. Equations (3.3) and (3.4) give $v_{1}^{+}-v_{1}^{-}=m\left(v_{2}^{+}-v_{2}^{-}\right)$; hence

$$
m\left(\gamma_{1}^{+}-\gamma_{1}^{-}\right)+\gamma_{2}^{+}-\gamma_{2}^{-}=0 .
$$

We have

$$
\gamma_{1}^{+}-\gamma_{1}^{-}=m Y_{t t} / \alpha(1-\alpha)+2 m_{t} Y_{t} / \alpha(1-\alpha)+B m_{\alpha} / y_{\alpha}-C m / y_{\alpha},
$$


where $C=\left[(Y / \alpha-y)_{t}\right]^{2} / \alpha+\left[(Y /(1-\alpha)+y)_{t}\right]^{2} /(1-\alpha)$, and $\gamma_{2}^{+}-\gamma_{2}^{-}=Y_{t t} /$ $\alpha(1-\alpha)-C / y_{\alpha}$. Using (7) gives

$$
Y_{t t} y_{\alpha}+2 m m_{t} Y_{t} y_{\alpha} /\left(1+m^{2}\right)-\alpha(1-\alpha) C+\alpha(1-\alpha) B m m_{\alpha} /\left(1+m^{2}\right)=0 .
$$

Thus (S1) and (S2) form a system of two equations in the unknown functions $x(t, \alpha), Y(t, \alpha)$. We seek particular solutions of this system, of the form

$$
x(t, \alpha)=\lambda(t)(2 \alpha-1), \quad y(t, \alpha)=\mu(t)(2 \alpha-1),
$$

with $\mu(t)>0$ for all $t>0$. Then we have

$$
\begin{gathered}
m=\lambda / \mu, \quad Y=-\alpha(1-\alpha) \mu, \\
v_{2}^{+}=(\alpha-1) \dot{\mu}, \quad v_{2}^{-}=\alpha \dot{\mu}, \\
v_{1}^{+}=-\alpha m \dot{\mu}+(2 \alpha-1) \dot{\lambda}, \quad v_{1}^{-}=(1-\alpha) m \dot{\mu}+(2 \alpha-1) \dot{\lambda}, \\
A=\left(\alpha^{3}+(1-\alpha)^{3}\right) \dot{\mu}^{2}, \quad B=(2 \alpha-1) \dot{\mu}^{2}, \quad C=\dot{\mu}^{2} .
\end{gathered}
$$

(S1) and (S2) yield the following system of second-order ordinary differential equations:

$$
\ddot{m}=m \dot{\mu}^{2} /\left(2 \mu^{2}\right), \quad 2 \mu \ddot{\mu}+4 \mu \dot{\mu} m \dot{m} /\left(1+m^{2}\right)+\dot{\mu}^{2}=0 .
$$

Substituting $\mu=\theta^{2 / 3}$, the second equation becomes $\left(\left(1+m^{2}\right) \dot{\theta}\right)^{\cdot}=0$, that is, $\dot{\theta}=c /\left(1+m^{2}\right.$ ), where $c$ is a positive constant (we are interested in the case where the layer is widening out). Introducing the function $\phi(\theta)$ which gives the angle of the sheets with the $x$-axis, $1 / m(t)=\tan \phi(\theta(t))$, the first equation becomes

$$
\phi^{\prime \prime}+(\sin 2 \phi) /\left(9 \theta^{2}\right)=0 .
$$

We are interested in the solutions of (3.6) satisfying $\phi(\theta)=a \theta^{1 / 3}+O(\theta)$ when $\theta$ goes to $0, a>0$ being a constant. If $\phi$ is a solution of (3.6), then for all $\alpha>0, \phi_{\alpha}(\theta)=\phi(\alpha \theta)$ gives another solution; consequently, it suffices to study the case $a=1$, which is considered in the following lemma.

LEMmA 2. There is a unique global solution of (3.6) such that $\phi(\theta)=\theta^{1 / 3}+O(\theta)$ when $\theta \rightarrow 0$. Also, there is a value $\theta^{*}>0$ such that $\phi$ is strictly increasing from 0 to $\pi$ on the interval $\left[0, \theta^{*}\right]$.

Proof. Let us define $\psi$ and $\sigma$ by $\tan \phi(\theta)=\theta^{1 / 3} \psi\left(\theta^{1 / 3}\right)$ and $\sigma=\theta^{1 / 3}$. Then $\phi$ satisfies (3.6) if and only if $\psi(\sigma)$ satisfies

$$
\psi^{\prime \prime}=2 \psi\left(\psi+\sigma \psi^{\prime}\right)^{2} /\left(1+\sigma^{2} \psi^{2}\right) .
$$

Equation (3.7) is nonsingular at $\sigma=0$, and the right member is locally Lipschitz in $\left(\psi, \psi^{\prime}\right)$; consequently, it admits a unique local solution satisfying $\psi(0)=1$, $\psi^{\prime}(0)=0$. This solution is strictly increasing on its whole interval of existence.

Since

$$
\operatorname{Arctan} x=x+O\left(x^{3}\right) \text { and } \quad \psi\left(\theta^{1 / 3}\right)=1+O\left(\theta^{2 / 3}\right),
$$

we have $\phi(\theta)=\theta^{1 / 3}+O(\theta)$ when $\theta$ goes to 0 .

This local solution uniquely extends into a global one, owing to the Lipschitz property of (3.6) (outside a neighborhood of 0 ). 
The uniqueness of this global solution comes from the uniqueness of the local solution of (3.7).

For the second point, we define $f(\sigma)=\phi\left(\sigma^{3}\right)$. Then (3.7) gives

$$
\left[\log \left(f^{\prime}\right)\right]^{\prime}=2 \psi^{\prime} /(\sigma \psi)^{\prime}=h(\sigma),
$$

where $h(\sigma) \geq 0$.

We denote $H(\sigma)=\int_{0}^{\sigma} h(s) d s$; noticing that $f^{\prime}(0)=1,(3.8)$ then yields

$$
f(\sigma)=\int_{0}^{\sigma} e^{H(s)} d s, \text { that is, } \phi(\theta)=\int_{0}^{\theta^{1 / 3}} e^{H(s)} d s .
$$

From this expression, we deduce that there is a value $\theta_{0}>0$ such that $\phi\left(\theta_{0}\right)=\pi / 2$. It implies that $\sigma \psi(\sigma)$ converges towards $+\infty$ when $\sigma$ goes to $\sigma_{0}=\theta_{0}^{1 / 3}$. Moreover, $\phi$ is obviously strictly increasing on $\left[0, \theta_{0}\right]$.

Now, let us see what happens for $\theta \geq \theta_{0}$. First we show that $\phi^{\prime}\left(\theta_{0}\right)>0$. Indeed, $\phi$ is increasing on $\left[0, \theta_{0}\right]$ and then $\phi^{\prime}\left(\theta_{0}\right) \geq 0$; furthermore we have $\phi^{\prime}\left(\theta_{0}\right) \neq 0$, because otherwise, using uniqueness, $\phi$ would be identical to the constant solution $\pi / 2$. From this we readily deduce that for $\theta \geq \theta_{0}, \phi$ is strictly increasing and $\phi^{\prime \prime} \geq 0$, as long as $\phi(\theta) \leq \pi$. Then we have $\phi(\theta) \geq \pi / 2+\left(\theta-\theta_{0}\right) \phi^{\prime}\left(\theta_{0}\right)$ and there is a value $\theta^{*}>\theta_{0}$ such that $\phi\left(\theta^{*}\right)=\pi$.

Let $\phi_{\alpha}$ be a solution of (3.6). The evolution of the system is given by the equation

$$
\dot{\theta}=c \sin ^{2} \phi_{\alpha}(\theta), \quad \theta(0)=0 .
$$

We write $\theta(t)=\xi(\alpha c t) / \alpha$, where now $\xi$ satisfies

$$
\dot{\xi}=\sin ^{2} \phi(\xi), \quad \xi(0)=0 .
$$

Since we have $\phi(\theta) \sim \theta^{1 / 3}$, we do not have uniqueness for Eq. (3.10). Indeed, on one hand we have the solution $\xi \equiv 0$, and on the other hand we have the solution $\xi(t)=F^{-1}(t)$, where $F$ is the strictly increasing function defined by

$$
F(\xi)=\int_{0}^{\xi} \frac{1}{\sin ^{2} \phi(s)} d s .
$$

This solution satisfies $\xi(t)^{1 / 3} \sim t / 3 \quad(t \rightarrow 0)$. We now have

LEMMA 3. $\lim _{t \rightarrow+\infty} \xi(t)=\theta^{*}$.

Proof. $\xi(t)$ is an increasing function; let us denote $\xi^{*}=\sup _{t>0} \xi(t)$. Then we have $\xi^{*} \leq \theta^{*}$; otherwise, there would exist a $t^{*}$ such that $\xi\left(t^{*}\right)=\bar{\theta}^{*}$; hence $\xi\left(t^{*}\right)=$ $\sin ^{2} \phi\left(\theta^{*}\right)=0$, which would imply $\xi(t) \equiv \theta^{*}$. If we now suppose that $0<\xi^{*}<0$, then $\sin ^{2} \phi\left(\xi^{*}\right)>0$. From this we deduce that $\xi(t) \geq$ constant $>0$ for large $t$; this implies $\xi^{*}=+\infty$. We can conclude that $\xi^{*}=\theta^{*}$.

Any solution of the considered family is defined by the functions of time:

$$
\begin{gathered}
\phi_{\alpha}(\theta(t))=\phi(\xi(\alpha c t)), \\
\mu(t)=\theta(t)^{2 / 3}=\alpha^{-2 / 3} \xi(\alpha c t)^{2 / 3} .
\end{gathered}
$$


Thus they can all be obtained from the particular solution $(\phi, \xi)$ by convenient changes of scales in time and space.

Now we compute the initial velocity profile corresponding to this solution. Let us suppose the profile is given by the function $k(y)$. We have

$$
u_{1}^{+}(t, \mu(t))=-k(\mu(t)) \quad \text { for all } t .
$$

Now $u_{1}^{+}(t, \mu(t))=-m \dot{\mu}+\dot{\lambda}=\dot{m} \mu$, where $\dot{m}=-\left(1+1 / \tan ^{2} \phi\right) \dot{\phi}, \dot{\phi}=\phi^{\prime}(\xi) \dot{\xi}$. It follows that

$$
k(\mu)=\mu \phi^{\prime}\left(\mu^{3 / 2}\right) .
$$

From the expression $k(\mu)=\frac{1}{3} e^{H(\sqrt{u})}$, we readily get

$$
k(\mu)=\frac{1}{3}+\frac{2 \mu}{3}+O\left(\mu^{3 / 2}\right) .
$$

The function $k(\mu)$ is strictly increasing on the interval $\left[0, \mu^{*}\right], \mu^{*}=\left(\theta^{*}\right)^{2 / 3}$. We see the velocity profile given by numerical computations in Figure 1.

Let us denote by $\mathbf{B}^{*}$ the layer $\left(-\mu^{*} \leq y \leq \mu^{*}\right)$. The above velocity profile gives a stationary solution of the Euler equations in $\mathbf{B}^{*}$ which we shall denote $\mathbf{u}_{0}$. Besides, we easily see that, for $t$ infinite, $\mu(t)$ and $m \mu(t)$ go to zero and $\lambda(t)$ converges towards $-k\left(\mu^{*}\right)$. Then we deduce that, for $|y| \leq \mu^{*}$,

$$
u_{2}^{ \pm}(t, y) \rightarrow 0 \quad \text { and } \quad u_{1}^{ \pm}(t, y) \rightarrow-y k\left(\mu^{*}\right) / \mu^{*}
$$

when $t \rightarrow+\infty$.

Finally, we state the main features of this example in the following proposition.

Proposition 3. There is an $x$-invariant solution of (F.E), admitting $\mathbf{u}_{0}$ for the initial value, continuous on $\mathbf{B}^{*}$ for all $t>0$, and consisting of a foliated layer which gradually widens. The width of the layer is equivalent to $t^{2} / 9$ (when $t$ goes to 0 ) and increases towards $\mathbf{B}^{*}$ as $t \rightarrow+\infty$, while the foliated flow asymptotes to a shear layer.

THIRD EXAMPLE. In this example, we consider a foliated flow consisting of two fluids of different densities $\rho^{+}, \rho^{-}$, subjected to a vertical gravity field $\mathbf{G}$. The system of equations describing the motion is easily obtained by slight changes in the system (F.E). Obviously Eqs. (1)-(5) are unchanged; we have only to replace (6) and (7) by

$$
\begin{gathered}
\rho^{+} e \gamma^{+}+\rho^{-}(1-e) \gamma^{-}=-\nabla p+\left(e \rho^{+}+(1-e) \rho^{-}\right) \mathbf{G}, \\
\left(\rho^{+}\left(\gamma^{+}-\mathbf{G}\right)-\rho^{-}\left(\gamma^{-}-\mathbf{G}\right)\right) \wedge \mathbf{n}=0 .
\end{gathered}
$$

We denote the system $(1) \cdots(5),\left(6^{\prime}\right),\left(7^{\prime}\right)$ by $(\text { F.E })_{g}$.

In the particular two-dimensional case where the sheets are vertical planes orthogonal to a vector $\mathbf{e}_{3}$ (we consider an orthonormal reference frame $\mathbf{e}_{1}, \mathbf{e}_{2}, \mathbf{e}_{3}$ ), the 
system reduces to a simpler form $\left(\mathrm{F} . \mathrm{E}^{\prime}\right)_{g}$ :

$$
\begin{aligned}
& e_{t}+\operatorname{div}\left(e \mathbf{u}^{+}\right)=0, \\
& \operatorname{div}\left(e \mathbf{u}^{+}+(1-e) \mathbf{u}^{-}\right)=0, \\
& \rho^{+} \gamma^{+}=-\nabla p+\rho^{+} \mathbf{G}, \\
& \rho^{-} \gamma^{-}=-\nabla p+\rho^{-} \mathbf{G} .
\end{aligned}
$$

Let us suppose that $\mathbf{G}=-g \mathbf{e}_{2}, g>0$, that the heavier fluid $F^{+}$initially occupies the region $y \geq 0$ (and $F^{-}$the region $y<0$ ), and that the initial velocities of the two fluids are equal to zero.

Of course $\left(\right.$ F.E $E_{g}^{\prime}$ always admits the trivial solution $\mathbf{u}^{+}=\mathbf{u}^{-}=0$. But we easily check that the functions $e, \mathbf{u}^{+}, \mathbf{u}^{-}$, and $p$, given in the layer $|y| \leq \alpha g t^{2} / 2$ by the formulas

$$
\begin{gathered}
\mathbf{u}^{+}(t, y)=\frac{y}{t}-\frac{\alpha g t}{2}, \quad \mathbf{u}^{-}(t, y)=\frac{y}{t}+\frac{\alpha g t}{2}, \\
e(t, y)=\frac{y}{\alpha g t^{2}}+\frac{1}{2}, \quad p(t, y)=-2 \frac{\rho^{+} \rho^{-}}{\rho^{+}+\rho^{-}} g y,
\end{gathered}
$$

where $\alpha=\left(\rho^{+}-\rho^{-}\right) /\left(\rho^{+}+\rho^{-}\right)$, give a solution of $(\text {F.E })_{g}$ corresponding to the same initial data. Of course $\mathbf{u}^{+}$(resp. $\mathbf{u}^{-}$) is continuously extended to the upper region (resp. lower) by the value 0 out of the layer, in the same way $e$ is extended by 1 in the upper region ( 0 in the lower) and $p$ by the hydrostatic pressure out of the layer.

Notice that this two-phase solution corresponds to the particles of $F^{+}$falling freely into the fluid $F^{-}$with the acceleration $\alpha g$, while the particles of $F^{-}$are moving with the acceleration $-\alpha g$.

4. Comments on the particular solutions of Sec. 3. 1. We shall not discuss the first example, which we consider to be mainly a curiosity.

2. By contrast, the second example gives, to our knowledge, the first explicit example of the nonuniqueness phenomenon arising in perfect fluid dynamics when we work with discontinuous velocity fields (vortex sheets). Here the Cauchy problem for (F.E) has two different solutions: the given stationary vortex sheet and the foliated solution.

Notice that nonuniqueness in Euler equations has been suspected for a long time. Prandtl's scenario, explaining the existence of irrotational steady flows with nonzero circulation around a body with a sharp edge, is based on the existence of a transient flow with vortex sheet, alternatively to the irrotational flow with zero circulation at each time. Rigorous proofs that such flows exist do not appear to have been given, although there is some experimental evidence about them.

Besides this point, the physical significance of this foliated solution is not obvious. Despite some efforts, we were not able to prove that it yields a linearly well-posed Cauchy problem. Thus the choice of the foliated solution instead of the steady vortex sheet can only rest on rather inconclusive arguments, like the fact that it is continuous for all $t>0$, or an analogy with Example 3 (see the comments below). 
Notice that this nonuniqueness phenomenon is classical in the study of the Cauchy problem for nonlinear hyperbolic systems, where we have a clear criterion (the entropy condition of Lax) to choose the good solution. Unfortunately (F.E) is not a hyperbolic system and this condition does not actually work. But Example 3 suggests to introduce some sort of generalized entropy condition (only heuristic at the present time) leading to prefer the foliated solutions (as the more chaotic ones). Besides, we can also notice from the examples that the foliated solutions increase (as times goes on) the functionals which are integrals of $f(e)$, for all concave functions $f$ on $[0,1]$ such that $f(0)=f(1)=0$; the particular case $f(e)=-e \log e-(1-e) \log (1-e)$ corresponds to the Boltzmann mixing entropy.

The foliated solutions that we have shown here in Examples 1 and 2 cannot be obtained as limits of sequences of viscous solutions, in the sense given by Di Perna and Majda [10]. Indeed if it were the case, as was proved by these authors, the sequence would converge strongly (in the $L^{2}$ sense) out of a space-time region of Hausdorff dimension $\leq 1$. In fact, if we want to consider foliated solutions as limits of sequences of viscous solutions with a vanishing viscosity, we certainly have to introduce a convenient vanishing noise also; the question is open, but we stress the fact that, in order to give realistic numerical simulations of shear layers, one has to introduce two small parameters, viscosity and noise [8].

If $\mathbf{u}_{0}$ is a given vortex sheet, is there a two-phase solution of (F.E) admitting $\mathbf{u}_{0}$ as initial data?

The answer is probably negative because of the well-known tendency of the vortex sheets to roll up into spirals [18]. It seems that only particular cases such as the one we have shown could relax in this "simple" way.

Working with vortex sheets, which are particular discontinuous solutions of Euler equations, leads to disconcerting observations: in two dimensions, we can prove [13] that there exists initial data for which there is a global solution for $t>0$ (which is an analytic vortex sheet), while we do not know anything about the existence of a solution for $t<0$, even in a weak sense, for such initial data. This is strange behavior for a Hamiltonian system.

3. In the third example, for the same initial conditions, we have two solutions: the trivial steady solution and the foliated one. But, in this case, we can show that the first solution corresponds to an ill-posed problem for the interface, while the second is linearly well-posed.

Let us consider first the well-known $[15,21]$ system of equations giving the evolution of the interface between the two fluids in irrotational motion.

Suppose the interface is given in the $(x, y)$ plane by the equation $y=f(t, x)$. Let $\tau(t, x)$ be the tangent unit vector to the interface at the point $(x, f(t, x))$, and $d \sigma$ the length element on the curve. The cinematic equation gives

$$
f_{t}=-f_{x} V_{1}+V_{2}
$$

and the dynamic equation gives

$$
\left(\rho^{+} \gamma^{+}-\rho^{-} \gamma^{-}\right) \cdot \tau=\left(\rho^{+}-\rho^{-}\right) \mathbf{G} \cdot \tau,
$$

where $\gamma^{+}$and $\gamma^{-}$are the accelerations of the particles of $F^{+}$and $F^{-}$which are 
at time $t$ at the position $(x, f(t, x))$, and $V_{1}$ and $V_{2}$ are the two components of the mean velocity $\mathbf{V}=\left(\mathbf{u}^{+}+\mathbf{u}^{-}\right) / 2$ on the interface; they are given by the following singular kernels:

$$
\begin{aligned}
& V_{1}(t, x)=-\frac{1}{2 \pi} f \frac{f(t, x)-f\left(t, x^{\prime}\right)}{\left(x-x^{\prime}\right)^{2}+\left(f(t, x)-f\left(t, x^{\prime}\right)\right)^{2}} \omega\left(t, x^{\prime}\right) d x^{\prime}, \\
& V_{2}(t, x)=\frac{1}{2 \pi} f \frac{x-x^{\prime}}{\left(x-x^{\prime}\right)^{2}+\left(f(t, x)-f\left(t, x^{\prime}\right)\right)^{2}} \omega\left(t, x^{\prime}\right) d x^{\prime},
\end{aligned}
$$

where $\omega(t, x)$ is the vorticity density on the curve, given by $\omega d x=-\left(\mathbf{u}^{+}-\mathbf{u}^{-}\right) \cdot \tau d \sigma$.

Taking $f_{x}(t, x)$ and $\omega(t, x)$ as unknown functions and linearizing the system about the solution $f_{x}=0, \omega=0$ gives straightforwardly the linear system:

$$
\left\{\begin{array}{l}
f_{x t}=\frac{1}{2} \Lambda \omega \\
\omega_{t}=2 \alpha g f_{x}
\end{array}\right.
$$

where the operator $\Lambda$ is defined, via the Fourier transform, by $\widehat{\Lambda \omega}(\xi)=2 \pi|\xi| \hat{\omega}(\xi)$ $(\hat{\omega}(\xi)$ is the Fourier transform of the function $\omega$ with respect to the $x$ variable).

This system yields $\hat{\omega}_{t t}=\alpha g 2 \pi|\xi| \hat{\omega}$, and we see that for $\alpha>0$ the Cauchy problem is ill-posed.

We can show, using the method of [13], that the nonlinear problem itself is illposed. Of course this problem can also be solved in an analytic setting, using the methods of $[15,21]$.

We come now to prove the assertion that the linearized system about the foliated solution is well-posed. We shall consider a perturbed initial state where the interface is nearly horizontal and the velocities are equal to zero. We shall carry through the computations only in the case where $\rho^{+}-\rho^{-}$is small compared to $\rho^{+}$. This amounts to saying that the two fluids have the same density (taken equal to 1 ) and $F^{+}$ is subjected to a field $\mathbf{G}$ while $F^{-}$is subjected to $-\mathbf{G}$. This introduces a symmetry which leads to noticeable simplifications in the calculations. The general case works as well, but the calculations are rather cumbersome.

Thus we start by considering the system:

$$
\begin{aligned}
& e_{t}+\operatorname{div}\left(e \mathbf{u}^{+}\right)=0, \\
& \operatorname{div}\left(e \mathbf{u}^{+}+(1-e) \mathbf{u}^{-}\right)=0, \\
& \gamma^{+}=-\nabla p+\mathbf{G}, \\
& \gamma^{-}=-\nabla p-\mathbf{G} .
\end{aligned}
$$

We easily check that this system implies that the quantities $e^{-1}$ curl $\mathbf{u}^{+}$(resp. $(1-e)^{-1}$ curl $\mathbf{u}^{-}$) are conserved when we follow the particles of $F^{+}$(resp. $F^{-}$). So, if we start with irrotational velocity fields $\mathbf{u}^{+}, \mathbf{u}^{-}$(this is obviously our case) they will remain irrotational at all times. This remark permits us to describe the system with only the two unknown functions $e(t, x, y)$ and $\phi(t, x, y)$, defined by $\mathbf{u}^{+}-\mathbf{u}^{-}=\nabla \phi$.

We note that

$$
\operatorname{curl} \overline{\mathbf{u}}=\nabla e \wedge \nabla \phi,
$$


and thus $\overline{\mathbf{u}}$ is given by the Biot-Savart formula:

$$
\overline{\mathbf{u}}(t, X)=-\frac{1}{2 \pi} \int \frac{X-X^{\prime}}{\left|X-X^{\prime}\right|^{2}} \wedge(\nabla e \wedge \nabla \phi) d X^{\prime}, \quad X=(x, y) .
$$

Then we get $\mathbf{u}^{+}, \mathbf{u}^{-}$by

$$
\mathbf{u}^{+}=\overline{\mathbf{u}}+(1-e) \nabla \phi, \quad \mathbf{u}^{-}=\overline{\mathbf{u}}-e \nabla \phi .
$$

Now, let us suppose that the foliated layer is parametrized by the function $y(t, \lambda, x)$. That is, $e(t, x, y(t, \lambda, x))=\lambda$.

The conservation equation then becomes

$$
y_{t}+y_{x} u_{1}-u_{2}-y_{\lambda} \operatorname{div}(e(1-e) \nabla \phi)=0 .
$$

Using the fact that curl $\mathbf{u}^{+}=\operatorname{curl} \mathbf{u}^{-}=0$, the dynamical equation can be written as

$$
\left(\mathbf{u}^{+}-\mathbf{u}^{-}\right)_{t}+\frac{1}{2} \nabla\left(\left|\mathbf{u}^{+}\right|^{2}-\left|\mathbf{u}^{-}\right|^{2}\right)=2 \mathbf{G} .
$$

Introducing the function $\Phi(t, \lambda, x)=\phi(t, x, y(t, \lambda, x))$, we obtain

$$
\Phi_{x t}-\left(\phi_{y} y_{t}\right)_{x}+\frac{1}{2}\left(\left|\mathbf{u}^{+}\right|^{2}-\left|\mathbf{u}^{-}\right|^{2}\right)_{x}+2 g y_{x}=0,
$$

where the subscripts $x, t$ indicate partial differentiation with $\lambda$ fixed.

An asymptotic expansion of the form

$$
\begin{aligned}
& y(t, \lambda, x)=y^{0}(t, \lambda)+\varepsilon y^{1}(t, x)+\varepsilon^{2} y^{2}(t, \lambda, x)+\cdots, \\
& \Phi(t, \lambda, x)=\Phi^{0}(t, \lambda)+\varepsilon \Phi^{1}(t, x)+\varepsilon^{2} \Phi^{2}(t, \lambda, x)+\cdots
\end{aligned}
$$

gives, for $t$ of the order of $\varepsilon$, the linearized equations (at the first order)

$$
\begin{aligned}
y_{t}^{1}-u_{2}^{1} & =0, \\
\Phi_{x t}^{1}+2 g y_{x}^{1} & =0,
\end{aligned}
$$

where $u_{2}^{1}$ is the first-order term in the asymptotic expansion of $u_{2}$ :

$$
u_{2}^{1}(t, \lambda, x)=-\frac{1}{2 \pi} \int_{0}^{1} d \lambda^{\prime} \int_{-\infty}^{+\infty} \frac{x-x^{\prime}}{\left(x-x^{\prime}\right)^{2}+\left(y^{0}(t, \lambda)-y^{0}\left(t, \lambda^{\prime}\right)\right)^{2}} \Phi_{x}^{1}\left(t, x^{\prime}\right) d x^{\prime} .
$$

We know $y^{0}(t, \lambda)=g t^{2}\left(\lambda-\frac{1}{2}\right)$, which is given by the unperturbed foliated solution (obtained by obvious changes in the formulas of Sec. 3, Example 3).

From the above integral, we get straightforwardly the estimate

$$
\left|u_{2}^{1}(t, \lambda, x)-u_{2}^{1}\left(t, \frac{1}{2}, x\right)\right| \leq \frac{g t^{2}}{8} \int_{-\infty}^{+\infty}\left|\Phi_{x x x}^{1}\right| d x^{\prime} .
$$

Then for $t \leq \varepsilon$, we get, at the first order, the linear system

$$
\begin{aligned}
& y_{t}^{1}-L(\theta)=0, \\
& \theta_{t}+2 g y_{x}^{1}=0,
\end{aligned}
$$

where $\theta=\Phi_{x}^{1}$ and $L(\theta)=u_{2}^{1}\left(t, \frac{1}{2}, x\right)$. 
Thus, we are led to solve the equation $\theta_{t t}=-2 g L(\theta)_{x}$. Taking the Fourier transform in the $x$ variable readily gives

$$
\hat{\theta}_{t t}(t, \xi)=\frac{2}{t^{2}}\left(1-\exp \left(-g t^{2} \pi|\xi|\right)\right) \hat{\theta}
$$

Since we consider the case where the interface is initially at rest $\left(\mathbf{u}^{+}=\mathbf{u}^{-}=0\right)$, we are interested only in the solutions such that $\theta(0, x)=0$. Then the real and imaginary parts of $\hat{\theta}$ can be bounded by the solutions of the equation

$$
\eta_{t t}=f(t, \xi) \eta
$$

where $f(t, \xi)=\inf \left(2 \pi g|\xi|, 2 t^{-2}\right)$. This gives

$$
|\hat{\theta}(t, \xi)| \leq \begin{cases}(2 \pi g|\xi|)^{-1 / 2} \sinh \left(t(2 \pi g|\xi|)^{1 / 2}\right)\left|\hat{\theta}_{t}(0, \xi)\right| & \text { for } t \leq(\pi g|\xi|)^{-1 / 2} \\ \left(a t^{2}|\xi|^{1 / 2}+b t^{-1}|\xi|^{-1}\right)\left|\hat{\theta}_{t}(0, \xi)\right| & \text { for } t>(\pi g|\xi|)^{-1 / 2}\end{cases}
$$

where $a, b$ are nonnegative constants.

From this we deduce that for a given $\theta_{t}(0, x)$ in the Sobolev space $H^{S}(\mathbb{R}) \quad(s$ is any real number), the solution $\theta(t, x)$ is, for all $t$, in the space $H^{S-1 / 2}(\mathbb{R})$.

Notice that the same features occur (ill-posed interface problem and existence of a two-phase relaxed solution) in the case of two fluids in a porous medium. But this last case falls into the frame of hyperbolic problems, and we can show that the steady flat interface is nonentropic in Lax's sense [16].

5. Conclusion. We have presented here a model of two-phase perfect fluid, based on the hypothesis that the local microstructure of the flow is a foliation of thin sheets. We have shown that this hypothesis yields a closed set of equations giving the evolution of the system. The evolution equation satisfied by the mean velocity has a supplementary term (Reynolds tensor) which can be explicitly calculated. This shows precisely the influence on the mean flow of this microstructure. It is of course a very particular "turbulent" situation where the local fluctuations of the velocity field are given by only two fields with probabilities $e$ and $1-e$. The Reynolds tensor does not look like some "eddy viscosity" term; it depends on the global structure of the flow and cannot be expressed only in terms of the mean velocity.

Example 3 shows us that this relaxed model permits us to transform an ill-posed interface problem into a linearly well-posed relaxed problem. This is probably not the case for Example 2, in which we were only able to show the nonuniqueness phenomenon hidden behind the ill-posedness of the Cauchy problem for vortex sheets. Nonuniqueness is due to the discontinuity of the initial velocity, since we have shown that, in the absence of external forces, a two-phase relaxation cannot occur with a regular initial condition.

Although it is problematic in Example 2, the relevance to physics of the two-phase solution given by Example 3 is quite reasonable. We may guess that it could be obtained as the limit of the solutions of Navier-Stokes equations with a vanishing viscosity and a small scale (high spatial frequency) periodic perturbation of the initial interface.

Acknowledgment. The authors thank the referee for his interesting suggestions and constructive criticism. 


\section{REFERENCES}

[1] V. Arnold, Chapitres Supplémentaires de la Théorie des Équations Différentielles Ordinaires, Mir, Moscow, 1984

[2] C. Bardos and U. Frisch, Finite time regularity for bounded and unbounded ideal incompressible fluids using Hölder estimates, Lecture Notes in Math. vol. 565, Springer-Verlag, 1975, pp. 1-14

[3] G. Birkhoff, Helmholtz and Taylor instability, Proc. Sympos. Appl. Math., vol. 13, Amer. Math. Soc., Providence, RI, 1962

[4] Y. Brenier, The least action principle and the related concept of generalized flows for incompressible perfect fluids, J. Amer. Math. Soc. 2, 225-255 (1989)

[5] R. Caflisch and O. Orellana, Long time existence for a slightly perturbed vortex sheet, Comm. Pure Appl. Math. 39, 807-838 (1986)

[6] R. Caflisch and O. Orellana, Singular solutions and well posedness for the evolution of vortex sheets, SIAM J. Math. Anal. 20, 293-307 (1989)

[7] J. R. Chan-Hong, Sur quelques problèmes à frontière libre en hydrologie, Thèse, Université de Lyon, 1987

[8] P. Comte, M. Lesieur, and J. P. Chollet, Simulation numérique d'un jet plan turbulent, C. R. Acad. Sci. Paris Ser. II Méc. Phys. Chim. Sci. Univers. Sci. Terre 305, 1037-1044 (1987)

[9] R. Di Perna, Measure-valued solutions to conservation laws, Arch. Rational Mech. Anal. 88, 223270 (1985)

[10] R. Di Perna and A. Majda, Reduced Hausdorff dimension and concentration-cancellation for twodimensional incompressible flow, J. Amer. Math. Soc. 1, 59-95 (1988)

[11] J. Duchon and R. Robert, Solutions globales avec nappe tourbillonnaire pour les équations d'Euler dans le plan, C. R. Acad. Sci. Paris Sér. I Math. 302, 183-186 (1986)

[12] J. Duchon and R. Robert, Elargissement diphasique d'une nappe tourbillonnaire en dynamique du fluide parfait incompressible, C. R. Acad. Sci. Paris Sér. I Math. 305, 701-704 (1987)

[13] J. Duchon and R. Robert, Global vortex sheet solutions of Euler equations in the plane, J. Differential Equations 73, 215-224 (1988)

[14] J. Duchon and R. Robert, Estimation d'opérateurs intégraux du type de Cauchy dans les échelles d'Ovsjannikov et applications, Ann. Inst. Fourier 36, 83-95 (1986)

[15] J. Duchon and R. Robert, Sur quelques problèmes à frontière libre analytique dans le plan, Séminaire Bony-Sjöstrand-Meyer, Exposé no. X, janvier 1985

[16] J. Duchon and R. Robert, to appear

[17] T. Kato, Non stationary flows of viscous and ideal fluids in $\mathbf{R}^{3}$, J. Funct. Anal. 9, 296-305 (1972)

[18] R. Krasny, Desingularization of periodic vortex sheet roll-up, J. Comp. Phys. 65, 292-313 (1986)

[19] D. W. McLaughlin, G. C. Papanicolaou, and O. R. Pironneau, Convection of microstructures and related problems, SIAM J. Appl. Math. 45, 780-797 (1985)

[20] D. Serre, Large oscillations in hyperbolic system of conservation laws, Prépublication, Université de St. Etienne, 1987

[21] C. Sulem, Thesis, University Paris-Nord, 1983

[22] C. Sulem, P. L. Sulem, C. Bardos, and U. Frisch, Finite time analyticity for the two and three dimensional Kelvin-Helmholtz instability, Comm. Math. Phys. 80, 485-516 (1981)

[23] L. C. Young, Lectures on the Calculus of Variations, Saunders, Philadelphia, 1969

[24] J. M. Delort, Existence de nappes de tourbillon pour l'équation d'Euler dans le plan, Prépublication, Université Paris-Sud (1990) 